\title{
Clinical and Videodermoscopic Evaluation of the Efficacy, Safety, and Tolerability of a Shampoo Containing Ichthyol, Zanthalene, Mandelic Acid, and Honey in the Treatment of Scalp Psoriasis
}

\author{
Maria Mariano $^{a}$ Maria Pia De Padova $^{b}$ Sandra Lorenzi ${ }^{c} \quad$ Norma Cameli $^{a}$

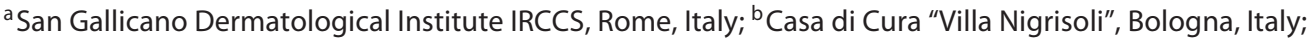 \\ 'Istituto Dermatologico Europeo, IDE, Milan, Italy
}

\section{Keywords}

Scalp · Psoriasis · Shampoo $\cdot$ Ichthyol $\cdot$ Zanthalene $\cdot$

Mandelic acid $\cdot$ Honey

\begin{abstract}
Purpose of the Study: The aim of the present prospective multicenter open study was to clinically and instrumentally evaluate the efficacy, safety, and tolerability of a shampoo, Mellis Cap $^{\circledR}$ shampoo, containing ichthyol, zanthalene, mandelic acid, and honeydew honey in the treatment of mild to moderate scalp psoriasis. Procedures: Thirty subjects with mild to moderate psoriasis applied the shampoo three times a week for 12 weeks. The outcome was evaluated at 30 days (T1), 60 days (T2), and 90 days (T3) of treatment, comparing it to baseline (T0) by means of clinical and patients' evaluation, digital photographs, and videodermoscopy. $\boldsymbol{R e}$ sults: Clinical and patients' evaluation showed improvement of scalp psoriasis and itching. This was confirmed by videodermoscopy analysis with a significant reduction of scalp psoriasis severity at T2 and T3 compared to baseline. No side effects were observed or reported. Conclusions:
\end{abstract}

\section{KARGER}

(c) 2018 S. Karger AG, Basel

E-Mail karger@karger.com

www.karger.com/sad
Study treatment was well tolerated and showed significant clinical and instrumental improvement of scalp psoriasis. Mellis Cap ${ }^{\circledR}$ shampoo is a good alternative to other medicated shampoos in the treatment of mild to moderate scalp psoriasis.

c) 2018 S. Karger AG, Basel

\section{Introduction}

Psoriasis is a chronic relapsing inflammatory skin disease characterized by abnormal keratinocyte hyperproliferation resulting in thickening of the epidermis and stratum corneum, vasodilatation, and increased permeability and inflammation. Scalp involvement is a common clinical feature of psoriasis. Up to $80 \%$ of individuals with psoriasis have scalp involvement, experiencing a negative impact on quality of life [1]. Scalp psoriasis is characterized by erythema and desquamation, sometimes associated with itching sensation. Videodermoscopy is a noninvasive diagnostic tool useful in the evaluation of body and scalp psoriasis that provides a magnified view of sur- 


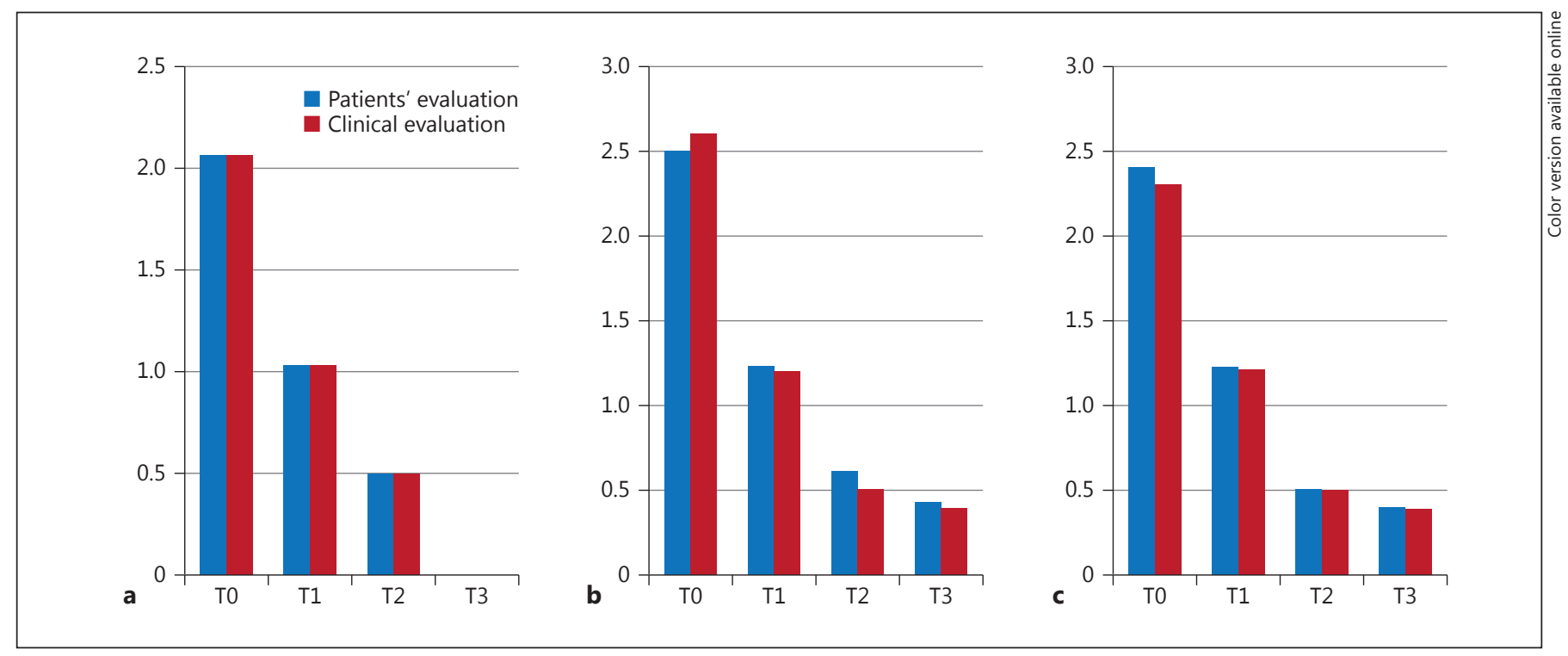

Fig. 1. Clinical and patients' evaluation of treatment for itching (a), erythema (b), and desquamation (c).

Fig. 2. Erythema and desquamation at baseline (T0) and at T3.
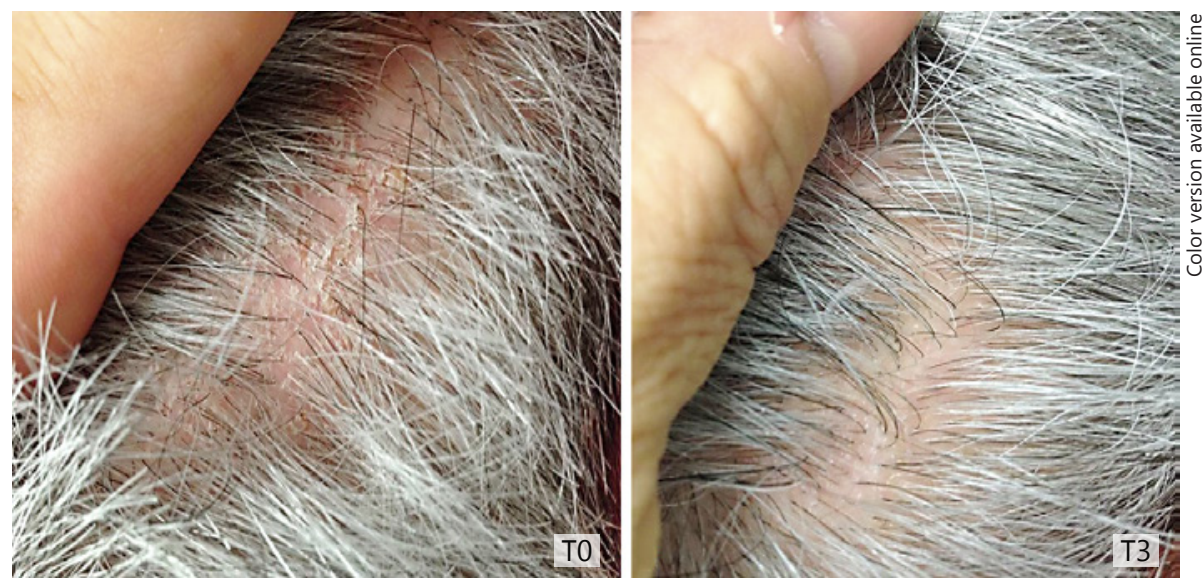

face components of epidermis and papillary dermis. The clinical outcomes of currently available shampoos for the treatment of scalp psoriasis are restricted, due to their limited efficacy, low cosmetic acceptance, and long-term safety and tolerability problems [2]. Effective and safe shampooing products are desirable to improve the longterm management of scalp psoriasis.

The aim of the present prospective multicenter open study was to clinically and instrumentally evaluate the efficacy, safety, and tolerability of a specific shampoo, Mellis Cap ${ }^{\circledR}$ shampoo, containing ichthyol, zanthalene, mandelic acid, and honeydew honey in the treatment of scalp psoriasis.

Ichthyol, Zanthalene, Mandelic Acid, and Honey Shampoo for Scalp Psoriasis

\section{Materials and Methods}

\section{Patients}

Thirty volunteer subjects with mild to moderate psoriasis, aged between 18 and 65 years ( 8 females and 7 males), were randomly enrolled in the study between December 2016 and January 2017. Informed consent was obtained from each patient. Inclusion criteria were as follows: mild to moderate scalp psoriasis, voluntary participation, preceding psoriasis therapies discontinued at least 6 months before the start of the study, and agreement not to use other shampoos during the study. Exclusion criteria were as follows: pregnancy, allergic contact dermatitis, current participation in another clinical study, any systemic psoriasis treatment within the past 6 months, UV phototherapy, and lack of cooperation. 

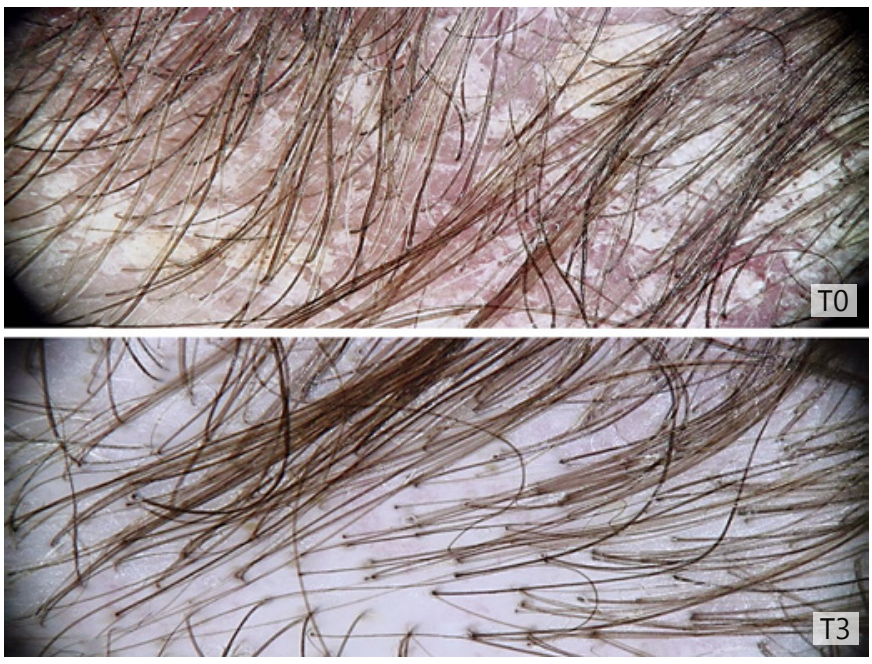

Fig. 3. Videodermoscopy image of erythema and desquamation at $\mathrm{T} 3$ compared to baseline (T0).
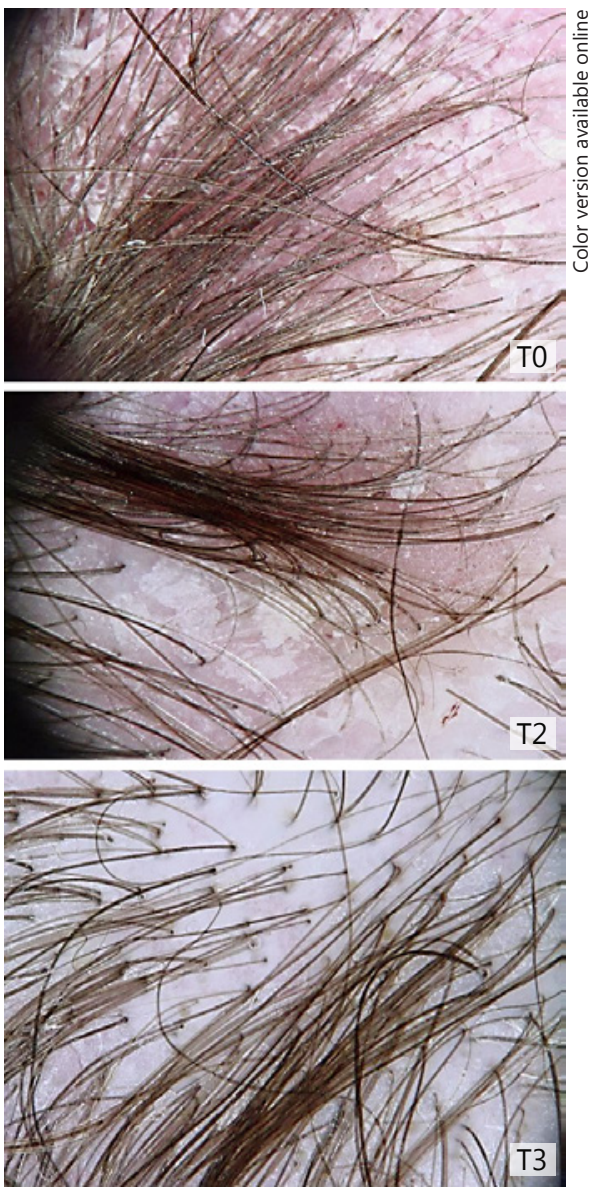

Fig. 4. T0: moderate scalp psoriasis; T2: mild improvement; T3: resolution of scaling.
Procedure

Patients applied the shampoo three times a week for 12 weeks and were instructed not to use any other shampoo or other topical drugs on the scalp. Each patient underwent a clinical dermatologic examination before (T0), 30 days (T1), 60 days (T2), and 90 days (T3) after the end of treatment to evaluate scalp psoriasis. At each visit, digital photographs were taken and dermoscopic images of the scalp were acquired using a dermoscope Trichoscan ${ }^{\circledR}$ (magnification 20-30-40-50-60-70×). Videodermoscopy features evaluated were erythema and desquamation (four-point scale: $0=a b$ sent, 1 = mild, 2 = moderate, 3 = severe). The clinical effect of treatment for itching, erythema, and desquamation was evaluated by investigators and patients' assessment (four-point scale: $0=a b$ sent, $1=$ mild, 2 = moderate, 3 = severe). The principles of the 1975 Declaration of Helsinki were followed.

\section{Statistical Analysis}

Statistical analysis was performed using nonparametric Wilcoxon test for paired samples because the sample data are not normally distributed.

\section{Results}

All the patients completed the 90-day treatment study. No side effects were observed or reported at the 30-day (T1), 60-day (T2), and 90-day (T3) follow-up visits. Clinical and patients' evaluation showed improvement of itching, erythema, and desquamation (Fig. 1a-c). At baseline, a moderate to severe itching was reported by $80 \%$ of patients. At the T3 visit, itching was absent in $100.00 \%$ of patients. The use of the shampoo significantly reduced erythema in $87.50 \%$ of patients and desquamation in $89.05 \%$ of patients (Fig. 1a, Fig. 2). At patients' evaluation at T3, scalp psoriasis was much improved in $84 \%$ of patients. These results were confirmed by a statistically significant decrease in erythema and desquamation at T2 and T3 compared to baseline at videodermoscopy analysis (Fig. 3-5). The treatment was safe and well tolerated: tolerability was good at $\mathrm{T} 1$ and high at $\mathrm{T} 2$ and T3 in $89 \%$ of patients.

\section{Discussion}

Scalp involvement is a common clinical aspect of psoriasis. Itching, erythema, and desquamation are typical features of scalp psoriasis. Eighty percent of those with scalp psoriasis experience a negative impact on quality of life [3] and long-term treatment is needed in most patients.

Despite a wide range of therapy options, scalp psoriasis remains difficult to treat and nonideal products are avail- 
Fig. 5. Clinical (a) versus videodermoscopy images (b) of mild scalp psoriasis at T0, completely resolved at the end of treatment (T3).
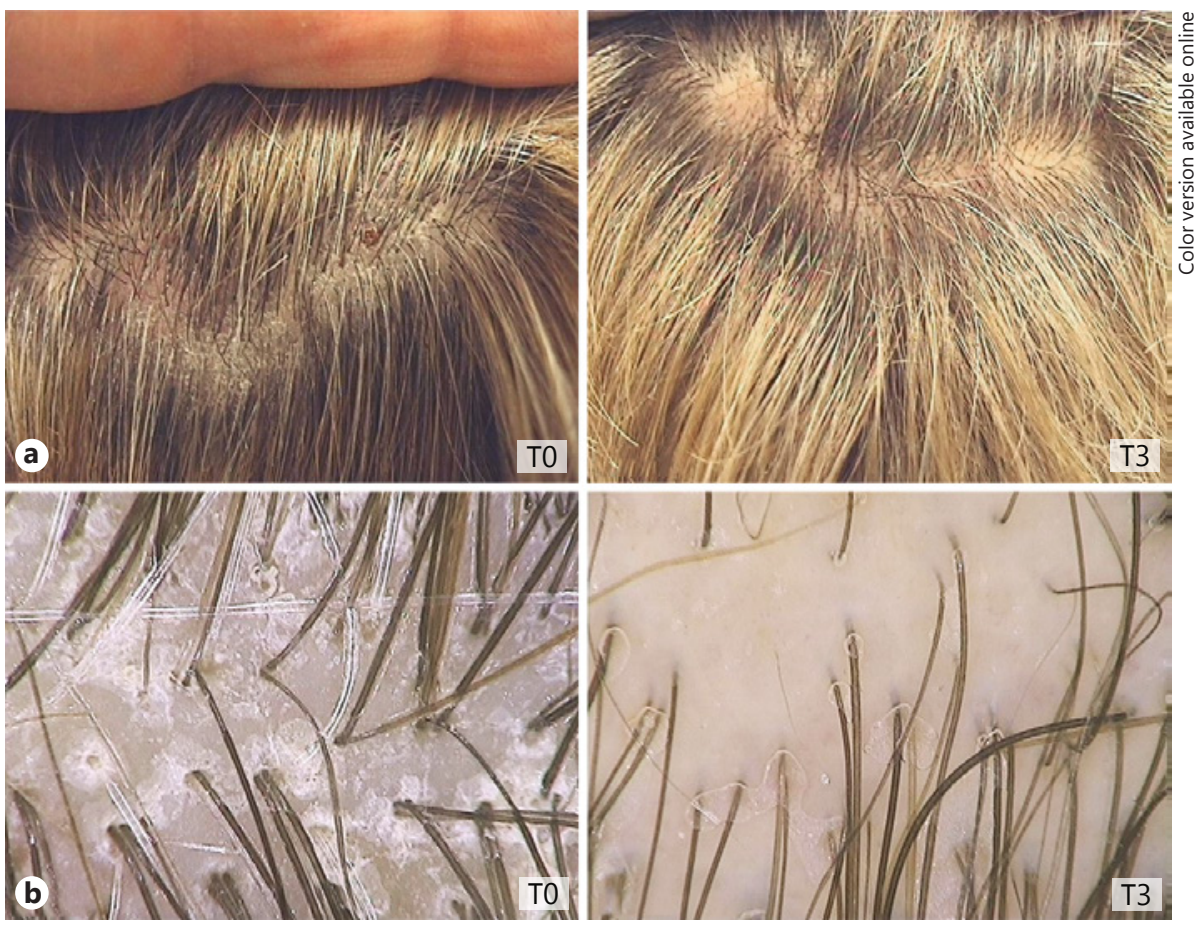

able. Topical treatment with corticosteroids with or without vitamin $\mathrm{D}_{3}$ analogues is the mainstay of treatment for scalp psoriasis [1]. However, long-term treatment could be limited by safety issues and by rebound problems when discontinued. Many topical treatments for scalp psoriasis are difficult or unpleasant to apply, resulting in decreased adherence and efficacy [4].

Patients' dissatisfaction supports the need for new, effective, and well-tolerated treatment options for scalp psoriasis. When the scalp is involved alone, aggressive treatments are not recommended. Shampoo is one of the most suitable formulations for topical therapy and management of scalp psoriasis [5]. Kobayashi et al. [6] found that proper hair washing improved scalp condition symptoms, and possibly the underlying etiology in patients with psoriasis; simple hair-washing techniques generally improved scalp conditions and decreased levels of the inflammatory markers IL-1 ra/IL-1 $\alpha$ in the horny layer [6].

In the choice of a shampoo for the treatment of scalp psoriasis, safety and efficacy of the ingredients, the reduction of recurrences, and sensitization should be considered. Corticosteroid-based shampoos could be effective but long-term use could be associated with safety and tolerability problems. Coal tar-based shampoos are not easy to use because of their low cosmetic acceptance.

Ichthyol, Zanthalene, Mandelic Acid, and Honey Shampoo for Scalp Psoriasis
In our study, we clinically and instrumentally evaluated the efficacy, safety, and tolerability of a shampoo, Mellis Cap ${ }^{\circledR}$ shampoo, containing ichthyol $1.2 \%$, mandelic acid $1.2 \%$, zanthalene $(<1 \%)$, and honeydew honey $(<1 \%)$ in the treatment of scalp psoriasis by means of clinical and patients' evaluation, digital photographs, and videodermoscopy. Ichtyol has an antiproliferative and anti-inflammatory activity inhibiting 5-lipoxygenase activity in human polymorphonuclear neutrophils [7]. Mandelic acid has well-known keratolytic and keratoplastic effects that help to remove the scales.

Zanthalene is a $\mathrm{CO}_{2}$ extract obtained from the fruit of Sichuan pepper, a Chinese spice known for its chemestetic properties with a strong anti-itching action [8]. Its alkylamides reduce skin sensitivity. In vitro tests showed a transitory synaptic transmission block which seems to be responsible for the marked anti-itching and anesthetic-like actions observed. Honeydew honey is prepared from secretions of plants or excretions of plant-sucking insects with important moisturizing, softening, and soothing virtues; it helps maintain soft and protected scalp and mitigates irritations [9].

The active compounds of Mellis Cap ${ }^{\circledR}$ shampoo provide an effective adjuvant treatment that can modulate scaling, itching, and inflammation, all characteristic features of scalp psoriasis. 


\section{Conclusion}

In this study, we have clinically and instrumentally analyzed the efficacy and safety of a shampoo formulation containing ichthyol, mandelic acid, zanthalene, and honeydew honey in the treatment of scalp psoriasis. Our results have shown that the study treatment is well tolerated and effective in controlling scaling and itching in mild to moderate scalp psoriasis. This could be a safe, tolerable, and effective alternative to other medicated shampoos in the treatment of mild to moderate scalp psoriasis and it can be associated with medical therapies in the treatment of severe scalp psoriasis.

\section{Statement of Ethics}

Informed consent was obtained from each patient.

\section{Disclosure Statement}

The authors declare that they have no competing financial interests or other potential conflict of interests. No funding sources were used for this article.

\section{References}

1 Guenther L: Current management of scalp psoriasis. Skin Therapy Lett 2015;20:5-7.

2 Grills CE, Cooper SM: Polidocanol: a potential contact allergen in shampoo. Contact Dermatitis 2007;56:178.

3 Krueger G, Koo J, Lebwohl M, Menter A, Stern RS, Rolstad T: The impact of psoriasis on quality of life: results of a 1998 National Psoriasis Foundation patient-membership survey. Arch Dermatol 2001;137:280-284.
-4 Papp K, Berth-Jones J, Kragballe K, Wozel G, de la Brassinne M: Scalp psoriasis: a review of current topical treatment options. J Eur Acad Dermatol Venereol 2007;21:1151-1160.

5 Crowley J: Scalp psoriasis: an overview of the disease and available therapies. J Drugs Dermatol 2010;9:912-918.

-6 Kobayashi M, Ito K, Sugita T, Murakami Y, Yamashita R, Matsunaka H, Nakamura M: Physiological and microbiological verification of the benefit of hair washing in patients with skin conditions of the scalp. J Cosmet Dermatol 2016;15:e1-e8.
7 Diezel W, Schewe T, Rohde E, Rosenbach T, Czarnetzki BM: Ammonium bituminosulfonate (Ichthyol). Anti-inflammatory effect and inhibition of the 5-lipoxygenase enzyme. Hautarzt 1992;43:772-774.

-8 Artaria C, Maramaldi G, Bonfigli A, Rigano L, Appendino G: Lifting properties of the alkamide fraction from the fruit husks of Zanthoxylum bungeanum. Int J Cosmet Sci 2011; 33:328-333.

9 Martinotti S, Calabrese G, Ranzato E: Honeydew honey: biological effects on skin cells. Mol Cell Biochem 2017;435:185-192. 